\section{INSULATING OILS}

$\mathrm{I}^{\mathrm{N}}$ a symposium on insulating oils, five important papers were presented before the Institution of Electrical Engineers in London on May 13 by various authors. In the first one, on "Manufacture and Testing of Oils and Oil-Rosin Saturants for use in Electrical Equipment", A. W. Thompson and J. C. Wood-Mallock described briefly the selection of oil crudes, and the choice of refinery methods best suited for the manufacture of transformer and switch oils, and condenser and cable saturants. Methods of test are discussed and consideration is given to the changes undergone by these liquid dielectrics under high electrical stresses.

Highly paraffinic oils are undesirable in transformers, cables and condensers, and therefore solvent processes are, in general, unsuited to the production of such oils. Rosin is a desirable constituent of cable saturants. It is concluded that in respect of transformer oils, revision of British Standard 148, particularly of the oxidation test, is overdue. Any proposed oxidation test should take account of acidity development as well as sludge, and should be carried out at a lower temperature. Solvent treatments lend themselves to production of transformer oils having the desired characteristics, but the distillate must be chosen for the purpose. For switch oils, further work is required on carbon production during arcing and its relation to refining.

For condenser saturants transformer oil is not ideal. They should be judged on the basis of dielectric loss and stability to ionic bombardment.

Sulphuric acid treatment of a naphthenic distillate, which may have a reasonably high viscosity, is the best method of production. In cable saturants for solid-type cables the oil component should be assessed on power loss, particularly at high temperatures, and freedom from gas formation under electrical stress. A suitable oil is prepared from a viscous vacuum distillate from a naphthenic or intermediate crude, by sulphuric acid refining. Solvent processes are unsuitable. Rosin is a desirable constituent because of its effect on gas formation and cable stability, and should be chosen with regard to its effect on dielectric loss.

The second paper, "Insulating Oils for Cables", by $\mathrm{S}$. Beckinsale, indicated the present position in Great Britain of the insulating oils and compounds available for cables, and described their behaviour in impregnating plants. The oils in question are of the paraffinic and naphthenic types, and either class may be used in oil-rosin compounds. Naphthenic oils are the better solvents for rosin and for the oxidized bodies produced from rosins, and their use is increasing rapidly. All the oils and compounds can be prepared with excellent electrical properties, but as some of their constituents are very subject to chemical change in the presence of air, the cablemaking plant and processes must be types which reduce this interaction to a minimum. For voltages up to and including $66 \mathrm{kv}$. the various oil-and-rosin compounds are employed, but for higher voltages either a thin naphthenic oil is used for the oil-filled. cables, or a viscous naphthenic oil, sometimes containing a viscous hydrocarbon polymer, is the saturant for the pressure-type cables.

Cable oils should be resistant to gassing, and both British and American oil refiners leave in certain aromatic constituents which absorb any gas that may be generated under electrical stress from the more saturated compounds in the oil. Solventrefined naphthenic fractions have been found suitable for cables, but the solvent-refined paraffinic fractions have proved unsuitable owing to their failure to absorb gas and to the physical instability of their rosin compounds. The use in Great Britain of oilrosin compounds for the impregnation of cables for voltages up to. $66 \mathrm{kv}$. is based on their increased viscosity combined with the excellent impregnation and good adhesion between compound and paper fibre, In the early 1920's, papers made from cellulose from the conifers were developed, for it was considered that as resinous substances had been present in the cells in the tree, such cellulose with its charac. teristic cellular structure would have a greater affinity for rosin than the cellulose produced from such fibres as manila and cotton. The use of this wood pulp has now become almost universal for power cables.

The further improvement of oils and rosins for cables is largely a problem for chemists and physicists, with electrical engineers in the background testing the results obtained from new processes and, where necessary, working out new methods of test as the dielectric losses are reduced.

The third paper of the series, by W. Fordham Cooper, on "Insulating Óil in Relation to CircuitBreaker Failures", is based upon the author's investi. gations in the course of the last ten years of a considerable number of accidents and dangerous occurrences due to the explosive failure of oil circuitbreakers and oil-immersed control gear, in the course of which it has become apparent that not all were due to inadequate rupturing capacity or defective insulation. This has been true particularly of a number of failures in recent years of the control gear for arc furnaces. The paper assesses what part deterioration of the switch oil may play, and the importance of the problem may be judged from the fact that oil in such switches is now frequently changed every two or four weeks, and, at some works, once a week.

Many engineers responsible for the maintenance of switchgear have felt that while the tests laid down in British Standard specifications may eliminate many new oils which are definitely unsuitable, they provide no certain guide to the rejection of used oil after a period of service. The author therefore includes a section suggesting the initial tests which might be taken in the search for a more suitable basis. Ex. plosions occur occasionally without previous insulation breakdown or severe arcing, and it is necessary to correlate their occurrences with some definable condition of the oil or the switch.

The study is divided into two parts : (i) surface contamination and the associated presence of suspended matter in the oil ; and (ii) general chemical deterioration of the oil. There are then four aspects of each of these, namely, reproduction under controlled conditions of the phenomena observed in the field ; laboratory examination of new and used oil, and correlation of the result with experience of breakdown; development of comparatively simple tests for the rejection of seriously defective oil ; the determination of whother repeatedly 'cleaning' oil by conventional methods renders it safe for re-use, or whether cumulative effects of chemical change may, after a time, lead to danger. In the initial stages the second and third aspects mentioned above must progress together so that, if possible, a corre- 
lation is established between simple tests and those requiring the services of a skilled chemist.

The next contribution is on "Mineral Oil for Transformers and Switchgear", by A. A. Pollitt, in which the author sets out to familiarize the electrical engineer with the nature and characteristics of mineral oils. In spite of the advent of non-inflam mable, liquid-chlorinated hydrocarbons, mineral oil still remains the chief dielectric used in liquid-filled transformers and switches. Mineral oil has been chosen because of its high electric strength compared with that of air and because, where necessary, it can function as a cooling medium and so maintain the operating temperature of the equipment at a safe value. The outstanding characteristic of mineral insulating oils is their stability in service. Efficient operation of mineral-oil-filled electrical equipment is dependent, therefore, to a large extent upon the ability of the oil to retain substantially unimpaired, in the presence of deteriorating influences, its initially good electrical properties, its inertness towards the solid insulating materials that are also present in most electrical equipment in which oil is used, and its ability to effect efficient cooling.

The author deals with the constitution of mineral insulating oils, refining, causes of deterioration, oxidation products and their influence, the evaluation of insulating oils, and oils in service.

The last paper, on the "Maintenance of Insulating Oils in the Field", by L. H. Welch, reminds one that insulating oil deteriorates in use and the maintenance engineer must see that the working conditions are such as to minimize this. The oil must be reconditioned when the necessity is revealed by regular observations on its condition. Reference is made to points which should be watched in the design and installation of plant and it is suggested that, of the oils specified in British Standard 148-1933, only grade $B .30$ should be retained. The revised specification should include high-viscosity oils which are frequently used in electrical apparatus. Routine tests for sludge and moisture contamination are universal, but determination of acid value may be important and the flash-point has to be determined occasionally. Suggestions are made as to what values of these should be permitted before purification is considered necessary. The type of contamination likely to be present and the apparatus available for its treatment and various methods of using it are discussed. A table showing suitable maintenance periods is given, and it is pointed out that as it is difficult to handle oil and to work on oil-immersed gear without causing deterioration, these periods depend more on the immersed apparatus requiring attention than on the oil.

Oil maintenance can be kept to a minimum by giving attention to the following points : minimum operating temperature of transformers and kindred apparatus to be ensured by adequate ventilation for indoor equipment and correct adjustments of controls on forced cooling systems; adequacy of breathers, and their maintenance; careful selection of mechanical and electrical design of apparatus, particularly in relation to tap-changing gear; use of grade $B$ oils-grade $A$ oils have proved liable to develop excessive acidity and sludging in service whereas grade $B$ oils have developed less total acidity and less total sludging and should, therefore, be used wherever oil of this viscosity is required; such oils can be expected to prove a reasonable cure for acidity and sludging, which have occurred where grade $A$ oils have previously been used; cold puri fication is much to be preferred and a filter is generally more useful and easier to operate than a centrifuge.

Finally, it is considered that the present British Standard 148 requires revision. It covers six oils of the same viscosity and these could all be replaced by $B .30$ oil. There is need to include other oils, how. ever, particularly a more viscous oil for switchgear.

\section{INSTITUTION OF ELECTRICAL ENGINEERS}

$\mathrm{T}$ HE report of the Council for the year 1941-42 of the Institution of Electrical Engineers discloses that 1,650 new members were elected during the year and that there are now 10,483 corporate members on the register. An Installations Section was inaugurated during the year with an initial membership of 1,127. There are now nine students' sections, aggregating a membership of 4,483 , which includes 1,944 graduates.

l.ost-war planning continues to receive attention and a joint committee has been set up consisting of two representatives of each of the three engineering institutions, while contact has also been made by the planning committee with a number of other organizations which are discussing planning problems. Co-operation with the Ministry of Labour and National Service in the working of the Electrical Engineering Section of the Central Register continues and 1,121 members have been placed on work of national importance. Reference is made to the Institution's part in the intensive training courses in engineering, which are designed to carry men from the Ordinary National Certificate to the Higher National Certificate standard in six months. Cooperation with the Institution of Mechanical Engineers has been promoted in this connexion.

More than two thousand members of all classes are on active service with the Forces, and arrangements have been made for ensuring that those liable for military service are posted to appropriate technical branches of the Forces. More than three hundred applicants for technical commissions in the Forces have been dealt with; two hundred cases were presented to the Beveridge Committee with the view of up-grading or transfer to duties in the Services more appropriate to the member's qualifications. More than 4,500 enrolments have been accepted by the War Office from men in the Army and the R.A.F. for tuition in various engineering courses under the scheme set up by the three engineering institutions, the Board of Education and the Service Departments.

Other activities upon which the Institution has been engaged are the preparation of codes of practice, war emergency standards, A.R.P. in the engineering industry, the statistical control of materials and manufactures, and fuel efficiency and economy. On the educational side, in collaboration with the Institutions of Civil and Mechanical Engineers, a joint panel of senior engineers has been formed for the purpose of delivering lectures to university engineering societies, in order to bring students into touch with actual practice. A centre of the Polish Society of Electrical Engineers, with headquarters in London, has been formed and the Institution's premises have been placed at its disposal for meetings, etc. 